\title{
Chemical oxidation of $N, N$-diethyl-m-toluamide by sulfate radical-based oxidation: kinetics and mechanism of degradation
}

\author{
K. S. Tay $・$ N. A. Rahman $\cdot$ M. R. Bin Abas
}

Received: 29 October 2011/Revised: 11 February 2012/ Accepted: 15 May 2012/Published online: 2 August 2012

(C) CEERS, IAU 2012

\begin{abstract}
A study was undertaken in order to understand the kinetics and mechanism of the chemical oxidation of $N, N$-diethyl- $m$-toluamide, an insect repellent, by sulfate radical. In this experiment, sulfate radical was generated using peroxymonosulfate with iron(II) and cobalt(II) as activator. The second-order rate constant for the reaction of the sulfate radical with $N, N$-diethyl- $m$-toluamide was found to be $(1.9 \pm 0.1) \times 10^{9} \mathrm{M}^{-1} \mathrm{~s}^{-1}$ at $\mathrm{pH} 7$ and a temperature of $25^{\circ} \mathrm{C}$. Experiments for the chemical oxidation of $N, N$-diethyl- $m$-toluamide in river water and secondary wastewater revealed that the percentage of the removal of $N, N$-diethyl- $m$-toluamide was slightly influenced by the water matrices. However, the selected oxidation systems are not effective for the removal of $N, N$-diethyl- $m$ toluamide in seawater. Transformation by-products of $N, N$-diethyl- $m$-toluamide generated using peroxymonosulfate/iron(II) sulfate, peroxymonosulfate/iron(II) chloride, peroxymonosulfate/cobalt(II) sulfate and peroxymonosulfate/cobalt(II) chloride systems were identified. The results indicated that there are slight differences in the distributions of the transformation by-products detected depending on the activator. The common transformation by-products detected in all selected oxidation systems are
\end{abstract}

Electronic supplementary material The online version of this article (doi:10.1007/s13762-012-0081-3) contains supplementary material, which is available to authorized users.

K. S. Tay $(\varangle) \cdot$ N. A. Rahman · M. R. Bin Abas Department of Chemistry, Faculty of Science, Environmental Research Group, University of Malaya, 50603 Kuala Lumpur, Malaysia

e-mail: khengsoo@um.edu.my
$N, N$-diethylbenzamide, $N$-ethyl- $m$-toluamide, $N, N$-dimethyl$m$-toluamide, $\quad N$-ethyl- $N$-acetyl- $m$-toluamide, 2-(diethylamino)-1- $m$-tolylethanone, monohydroxylated $N$-ethyl- $m$ toluamide, and dihydroxylated $N, N$-diethyl- $m$-toluamide. Monohydroxylated $N, N$-diethyl- $m$-toluamide were detected only when the peroxymonosulfate/iron(II) system was applied. For the peroxymonosulfate/cobalt(II) systems, additional isomers of monohydroxylated $N$-ethyl- $m$-toluamide were detected. Peroxymonosulfate/cobalt(II) also transformed 2-(diethylamino)-1- $m$-tolylethanone into $N$-ethyl$N$-(2-oxo-2- $m$-tolylethyl)acetamide and monohydroxylated 2-(ethyl(vinyl)amino)-1- $m$-tolylethanone as transformation by-products.

Keyword Advanced oxidation processes - Kinetics . Mechanism - Peroxymonosulfate - Monosulfate

\section{Introduction}

Advanced oxidation processes (AOPs) involving the hydroxyl radical $(\cdot \mathrm{OH})$ have been considered as an important technology for the remediation of recalcitrant organic pollutants in industrial wastewater, municipal wastewater (Stasinakis 2008) and surface water (Weinberg et al. 2008). In fact, $\cdot \mathrm{OH}$ can react with almost all organic compounds but it also can be inactivated by the water matrices due to its non-selective behavior (e.g. Vahabzadeh 2005; Mckay et al. 2011; Matta et al. 2011). Possessing a redox potential of $2.6 \mathrm{~V}$, the sulfate radical $\left(\mathrm{SO}_{4}{ }^{--}\right)$has been reported to be more selective than $\cdot \mathrm{OH}$, as it reacts preferably with organic pollutants via an electron transfer reaction. $\mathrm{SO}_{4}^{-}$can also react with pollutants through hydrogen abstraction and addition reaction (Anipsitakis et al. 2006). Manoj et al. (2007) revealed that cyanuric 
acid, an end product derived as a result of $\cdot \mathrm{OH}$-mediated atrazine degradation, can be effectively removed using $\mathrm{SO}_{4}{ }^{-}$. Consequently, $\mathrm{SO}_{4}{ }^{-\cdot}$ is expected to oxidize a broader range of organic pollutants. Since the degradation mechanisms of organic pollutants via $\mathrm{SO}_{4}{ }^{-}$-based oxidation are different from $\cdot \mathrm{OH}-m e d i a t e d$ oxidation, the effectiveness of $\mathrm{SO}_{4}{ }^{-}$-based oxidation in treating various organic pollutants with a great diversity of molecular structure and chemical properties should be evaluated (Nfodzo and Choi 2011). So far, the treatment of organic pollutants such as atrazine (Chan and Chu 2009), pharmaceuticals and personal care products (e.g. Chan et al. 2010; Matta et al. 2011; Nfodzo and Choi 2011; Rickman and Mezyk 2010), methyl tert-butyl ether (Liang et al. 2010), polycyclic aromatic aromatic hydrocarbons (Wang et al. 2008), phenols (e.g. Anipsitakis et al. 2006; Yang et al. 2008), and so on using $\mathrm{SO}_{4}{ }^{-\cdot}$ has been reported.

This study was undertaken in order to assess the feasibility of $\mathrm{SO}_{4}{ }^{-\cdot}$ in the degradation of DEET ( $N, N$-diethyl- $m$ toluamide). DEET is an active compound in most ticks and insect repellents which functions as a blocker to insect's chemoreceptor that senses carbon dioxide and lactic acid in locating their host (Fradin 1998). Due to its wide use, contaminations of DEET in aquatic environment such as streams (Kolpin et al. 2004), seawater (Weigel et al. 2002, 2004), sewage plant effluents (Weigel et al. 2004), and even drinking water (Stackelberg et al. 2004) have been extensively reported. Although the removal of DEET by ozonation (Tay et al. 2009), photoinduced processes (Medana et al. 2010), and anodic Fenton treatment (Zhang and Lemley 2006, 2007) has been reported, the treatment of DEET by $\mathrm{SO}_{4}{ }^{-\cdot}$ has not been evaluated. Therefore, the objectives of this work are (i) to evaluate the influence of operating parameters on the chemical oxidation of DEET using $\mathrm{SO}_{4}{ }^{-}$; (ii) to evaluate the efficiency of $\mathrm{SO}_{4}{ }^{-\cdot}$ in the removal of DEET in river water, secondary wastewater and seawater; (iii) to determine the absolute rate constant for the reaction between DEET with $\mathrm{SO}_{4}^{-}$; and (iv) to identify the major transformation by-products and to propose the mechanisms for the DEET transformation during $\mathrm{SO}_{4}{ }^{-}$based oxidation. In this study, $\mathrm{SO}_{4}^{-\cdot}$ was generated using cobalt(II) $\left(\mathrm{Co}^{2+}\right)$ - and iron(II) $\left(\mathrm{Fe}^{2+}\right)$-mediated activation of peroxymonosulfate (PMS). This research has been carried out in the Department of Chemistry, Faculty of Science, University of Malaya (Kuala Lumpur; Malaysia), from August 2010 to February 2011.

\section{Materials and methods}

DEET (97\%), $p$-toluic acid (98\%) and $m$-toluic acid (99\%) were obtained from Aldrich (USA). Phenol $(\geq 99.5 \%)$ and formic acid $(98 \%)$ were purchased from
Fluka (Germany). Iron(II) chloride tetrahydrate $\left(\mathrm{FeCl}_{2}\right.$. $\left.4 \mathrm{H}_{2} \mathrm{O}\right)(>99 \%)$, iron(II) sulfate heptahydrate $\left(\mathrm{FeSO}_{4}\right.$. $\left.7 \mathrm{H}_{2} \mathrm{O}\right)(99.5 \%)$, cobalt(II) sulfate heptahydrate $\left(\mathrm{CoSO}_{4}\right.$. $\left.7 \mathrm{H}_{2} \mathrm{O}\right)(>99 \%)$, cobalt(II) chloride hexahydrate $\left(\mathrm{CoCl}_{2}\right.$. $\left.6 \mathrm{H}_{2} \mathrm{O}\right)(99 \%)$, cobalt(II) nitrate hexahydrate $\left(\mathrm{Co}\left(\mathrm{NO}_{3}\right)_{2}\right.$. $\left.6 \mathrm{H}_{2} \mathrm{O}\right)(99 \%)$ and potassium peroxymonosulfate (extra pure, minimum $4.5 \%$ active oxygen) were purchased from Acros Organics (USA). All aqueous solutions were prepared using ultrapure water (Elga, USA). Solvents, sulfuric acid and hydrochloric acid were purchased from Merck (Germany). All solvents were of high-performance liquid chromatography (HPLC) grade and were used without purification. Phosphate buffer $(0.5 \mathrm{M})$ was prepared using sodium dihydrogen-phosphate (Aldrich, USA) and disodium hydrogen-phosphate (Riedel-de Haën, Germany) and the $\mathrm{pH}$ was adjusted using either phosphoric acid (Merck, Germany) or sodium hydroxide (Fluka, Germany) solutions.

\section{Chemical oxidation of DEET in water}

All experiments were carried out in batch mode. Experiments were conducted in a $100 \mathrm{~mL}$ conical flask at room temperature $\left(25-28{ }^{\circ} \mathrm{C}\right)$. For the influence of operating parameters, the experiments were performed in ultrapure water. Reactions were initiated by the addition of PMS solution to the DEET solution containing $\mathrm{Fe}^{2+}$ or $\mathrm{Co}^{2+}$ as activator in a $100 \mathrm{~mL}$ conical flask. The solutions were stirred throughout the experiments using a magnetic stirrer. $1 \mathrm{~mL}$ of the aliquot was withdrawn at defined time intervals and the reaction was quenched immediately using 100 $\mu \mathrm{L}$ of methanol. The remaining concentration of DEET was determined using HPLC. The details of the experimental conditions are presented in Fig. 1.

For the effect of water matrices, the chemical oxidation of DEET was performed in river water, seawater and secondary wastewater. For these experiments, PMS were activated using $\mathrm{CoSO}_{4}$. The selected initial concentrations of PMS $\left([\mathrm{PMS}]_{0}\right), \mathrm{Co}^{2+}$, and DEET $\left([\mathrm{DEET}]_{0}\right)$ were of 250,25 and $25 \mu \mathrm{M}$, respectively.

Rate constant determination

The reaction rate constant for the reaction of DEET with $\mathrm{SO}_{4}{ }^{-\cdot}, \mathrm{k}_{\mathrm{SO}_{4}^{-}}$(DEET) was determined using the competition kinetics method and benzene was selected as the reference compound (Matta et al. 2011; Méndez-Díaz et al. 2010). The reported reaction rate constant for benzene with $\mathrm{SO}_{4}{ }^{-}$. ( $\mathrm{k}_{\mathrm{SO}_{4}-}$ (benzene)) was $2.4 \times 10^{9} \mathrm{M}^{-1} \mathrm{~s}^{-1}$ (Ziajka and Pasiuk-Bronikowska 2005). The reaction was performed at $25{ }^{\circ} \mathrm{C}$ in a $100 \mathrm{~mL}$ jacketed beaker containing DEET, benzene and $\mathrm{CoSO}_{4}$, each at a concentration of $25 \mu \mathrm{M}$. pH 

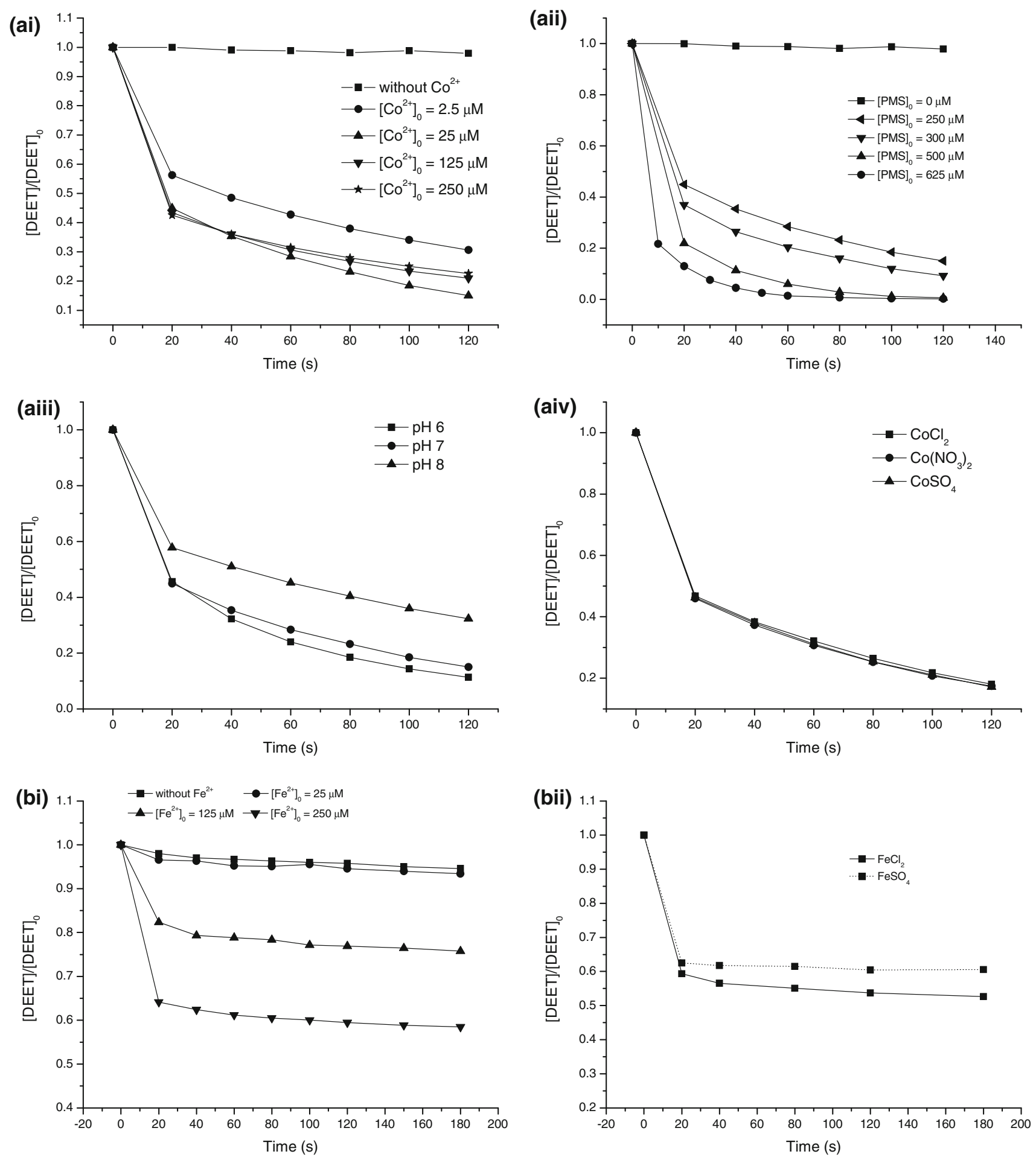

Fig. 1 Chemical oxidation of DEET using $\mathbf{a} \mathrm{Co}^{2+}$-PMS system [effect of (ai) $\mathrm{Co}^{2+}$ concentration $\quad\left([\mathrm{PMS}]_{0}=250 \mu \mathrm{M} ; \quad \mathrm{pH}=7\right.$; $\left.[\mathrm{DEET}]_{0}=25 \mu \mathrm{M}\right)$, (aii) PMS doses $\left([\mathrm{PMS}]_{0}:\left[\mathrm{Co}^{2+}\right]_{0}=100: 1\right.$; $\left.\mathrm{pH}=7 ;[\text { DEET }]_{0}=25 \mu \mathrm{M}\right),($ aiii $) \mathrm{pH}\left([\mathrm{PS}]_{0}=250 \mu \mathrm{M} ;\left[\mathrm{Co}^{2+}\right]_{0}=\right.$ $\left.25 \mu \mathrm{M} ;[\mathrm{DEET}]_{0}=25 \mu \mathrm{M}\right)$, and (aiv) counteranions $\left([\mathrm{PMS}]_{0}=\right.$

$\left.\left.250 \mu \mathrm{M} ; \quad\left[\mathrm{Co}^{2+}\right]_{0}=25 \mu \mathrm{M} ; \quad \mathrm{pH}=7 ; \quad[\mathrm{DEET}]_{0}=25 \mu \mathrm{M}\right)\right] \quad$ and (b) $\mathrm{Fe}^{2+}$-PMS system [effect of (bi) $\mathrm{Fe}^{2+}$ concentration $\left([\mathrm{PMS}]_{0}=250 \mu \mathrm{M} ; \quad \mathrm{pH}=7 ; \quad\right.$ Activator $=\mathrm{FeSO}_{4} ; \quad[\mathrm{DEET}]_{0}=$ $25 \mu \mathrm{M})$ and (bii) counteranions $\left([\mathrm{PMS}]_{0}=250 \mu \mathrm{M} ; \mathrm{pH}=7\right.$; $\left.\left.[\text { DEET }]_{0}=25 \mu \mathrm{M}\right)\right]$ 
was adjusted to 7 using $50 \mathrm{mM}$ of phosphate buffer. The reaction was started by the addition of $250 \mu \mathrm{M}$ of PMS solution, and the final volume of the reaction mixture was $100 \mathrm{~mL} .1 \mathrm{~mL}$ of the aliquot was withdrawn every $10 \mathrm{~s}$, and the reaction was quenched immediately using $100 \mu \mathrm{L}$ of methanol. $100 \mu \mathrm{L}$ of the reaction mixture was then injected into the HPLC for the determination of the remaining target compound and reference compound. The rate constant was calculated using the following equation:

$\ln \left(\frac{[\text { DEET }]_{t}}{[\text { DEET }]_{0}}\right)=\frac{\mathrm{k}_{\mathrm{SO}_{4}^{-}}(\text {DEET })}{\mathrm{k}_{\mathrm{SO}_{4}^{-}}(\text {benzene })} \ln \left(\frac{[\text { benzene }]_{t}}{[\text { benzene }]_{0}}\right)$

This competition kinetics method was validated using $p$ toluic acid, $m$-toluic acid, and phenol (Table 1).

Determination of the transformation by-products

The transformation by-products of DEET generated using PMS activated with $\mathrm{FeSO}_{4}, \mathrm{FeCl}_{2}, \mathrm{CoSO}_{4}$, and $\mathrm{CoCl}_{2}$ were evaluated. The initial concentration of PMS was set as $250 \mu \mathrm{M}$. The ratios of $[\mathrm{PMS}]_{0}:\left[\mathrm{Co}^{2+}\right]_{0}$ and $[\mathrm{PMS}]_{0}:\left[\mathrm{Fe}^{2+}\right]_{0}$ were set at 10:1 and 1:2, respectively. The reaction was quenched at a reaction time of 2 min with $5 \mathrm{~mL}$ of methanol. The entire reaction mixture was then extracted using the liquid-liquid extraction method with dichloromethane as the extracting solvent. The extracts were dried by evaporation and then dissolved in $250 \mu \mathrm{L}$ of dichloromethane. A $1.0 \mu \mathrm{L}$ aliquot of the extract was injected into GCMS.

Instrumental analysis

The details of the instrumental analysis were given in a previous study (Tay et al. 2010). All HPLC analyses were performed using Shimadzu HPLC system consisted of a LC-20AT pump, a SPD-M20A diode array detector, a SIL20AHT auto sampler, a CTO-20AC column oven and a

Table 1 Rate constants for reaction of $\mathrm{SO}_{4}{ }^{-\cdot}$ with $p$-toluic acid, $m$ toluic acid, phenol and DEET

\begin{tabular}{lll}
\hline Compound & \multicolumn{2}{l}{ Rate constant $\left(\mathrm{M}^{-1} \mathrm{~s}^{-1}\right)$} \\
\cline { 2 - 3 } & Literature & Experimental \\
\hline$p$-Toluic acid & ${ }^{\mathrm{a}} 1.8 \times 10^{9}$ & $(1.6 \pm 0.2) \times 10^{9}$ \\
$m$-Toluic acid & ${ }^{\mathrm{a}} 2.0 \times 10^{9}$ & $(1.5 \pm 0.2) \times 10^{9}$ \\
Phenol & ${ }^{\mathrm{b}} 8.8 \times 10^{9}$ & $(8.1 \pm 0.3) \times 10^{9}$ \\
DEET & - & $(\mathbf{1 . 9} \pm \mathbf{0 . 1}) \times \mathbf{1 0}^{9}$
\end{tabular}

${ }^{a}$ Neta et al. (1977)

b Ziajka and Pasiuk-Bronikowska (2005)

- Not available
CBM-20A communication bus module (Shimadzu, Japan). A reversed-phase Chromolith RP-18 monolithic column (100 mm $\times 4.6 \mathrm{~mm}$; Merck, Germany) was used for separation. The mobile phase used was a mixture of acetonitrile (Solvent A) and $0.1 \%$ formic acid in ultrapure water (Solvent B). The flow rate was maintained at

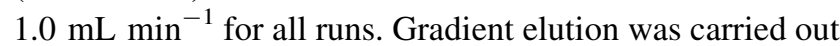
as follows: the initial mobile phase was a mixture of 20A:80B (v/v); subsequently, it increased to $80 \mathrm{~A}: 20 \mathrm{~B}(\mathrm{v} / \mathrm{v})$ over a period of $8 \mathrm{~min}$ and maintained at this ratio for 2 min. A 6 min reequilibration using 20A:80B (v/v) was carried out between sample injections. The detection wavelength was $200 \mathrm{~nm}$.

The analysis of transformation by-products was carried out using a Shimadzu GCMS system (Model: GCMSQP2010-Plus) equipped with an Rtx-5MS (Crossbond $5 \%$ diphenyl/95 \% dimethyl polysiloxane) column of dimensions $30 \mathrm{~m} \times 0.25 \mathrm{~mm} \times 0.25 \mu \mathrm{m} \quad$ (Restek, USA). Helium (purity $99.999 \%$ ) was used as the carrier gas. The average velocity of carrier gas was set at $40.2 \mathrm{~cm} / \mathrm{s}$. The temperature for the GC oven was programmed for an initial temperature of $60{ }^{\circ} \mathrm{C}$, maintained for $2 \mathrm{~min}$, which was further increased to $280{ }^{\circ} \mathrm{C}$ at the rate of $6{ }^{\circ} \mathrm{C} \mathrm{min}{ }^{-1}$ and was maintained at this temperature for $2 \mathrm{~min}$. The temperatures of the injection port and the transfer line were maintained at 300 and $310^{\circ} \mathrm{C}$, respectively. The data for quantitative analysis were acquired in the electron impact mode $(70 \mathrm{eV})$, scanning in the range of 50 to 700 atomic mass units (amu).

\section{Results and discussion}

Degradation of DEET using PMS activated by iron (II) and cobalt (II) ions

The generation of $\mathrm{SO}_{4}{ }^{-\cdot}$ using the combination of PMS with $\mathrm{Co}^{2+}$ and $\mathrm{Fe}^{2+}$ as activators was in accordance with the following simplified reactions (Chan and Chu 2009; Anipsitakis et al. 2006; Rastogi et al. 2009):

$\mathrm{HSO}_{5}^{-}+\mathrm{Co}^{2+} \rightarrow \mathrm{SO}_{4}^{-\cdot}+\mathrm{Co}^{3+}+\mathrm{HO}^{-}$
$\mathrm{HSO}_{5}^{-}+\mathrm{Fe}^{2+} \rightarrow \mathrm{SO}_{4}^{-}+\mathrm{Fe}^{3+}+\mathrm{HO}^{-}$

Figure 1 shows the influence of various operating parameters in the removal of DEET using PMS. A faster and more efficient method of DEET removal was observed when $\mathrm{Co}^{2+}$ concentration increased from 2.5 to $25 \mathrm{mM}$ (Fig. 1ai). A further increase in $\mathrm{Co}^{2+}$ concentration did not show any significant improvement in the removal of DEET. This is most likely due to the oxidation of $\mathrm{Co}^{2+}$ to $\mathrm{Co}^{3+}$, which is a side reaction that consumes a part of the $\mathrm{SO}_{4}{ }^{-\cdot}$ as indicated in reaction (4) (Anipsitakis et al. 2006). 
$\mathrm{SO}_{4}^{-\cdot}+\mathrm{Co}^{2+} \rightarrow \mathrm{SO}_{4}^{--}+\mathrm{Co}^{3+}$

Figure 1aii shows that on increasing the $[\mathrm{PMS}]_{0}$, the efficiency of DEET removal was also increased. It is shown that doses of PMS corresponding to the ratio of $[\mathrm{PMS}]_{0}$ to [DEET] $]_{0}$ of 20:1 and 25:1 led to $90 \%$ of DEET degradation at 120 and $80 \mathrm{~s}$ of reaction time, respectively. The efficiency of DEET removal using selected oxidation systems was $\mathrm{pH}$ dependence (Fig. 1aiii). The removal of DEET at basic condition was found to be lower than that of the acidic and neutral condition. At $\mathrm{pH} 8$, the rate of DEET removal was slightly retarded due to the precipitation of $\mathrm{Co}^{2+}$ as $\mathrm{Co}(\mathrm{OH})_{2}$, and it reduced the amount of $\mathrm{Co}^{2+}$ available for the activation of PMS (Chan and Chu 2009). The influence of counteranions for the DEET removal was performed using nitrate, sulfate and chloride salts of $\mathrm{Co}^{2+}$. The results indicated that there is no significant influence from the selected counteranions for the DEET removal (Fig. 1aiv) when $\mathrm{Co}^{2+}$ is selected as activator.

DEET removal was also performed using $\mathrm{FeSO}_{4}$ and $\mathrm{FeCl}_{2}$ as activators (Fig. 1b). Fig 1 bi shows that at the $\mathrm{Fe}^{2+}$ concentration of $250 \mu \mathrm{M}$ which correspond to the ratio of $[\mathrm{PMS}]_{0}:\left[\mathrm{Fe}^{2+}\right]_{0}$ of $1: 1$, only $42 \%$ of DEET removal was achieved. Therefore, $\mathrm{Co}^{2+}$ is a better activator for PMS compared with $\mathrm{Fe}^{2+}$. Using $\mathrm{Fe}^{2+}$ as activator, the rate of DEET removal was also found to be influenced by the presence of counteranions (Fig. 1bii). The presence of the chloride ion slightly enhanced the rate of DEET degradation. It might be due to the oxidation of the chloride ion for the formation of the chloride radical which can react with DEET and, hence, accelerated the rate of DEET degradation (Anipsitakis et al. 2006).

\section{Chemical oxidation of DEET in different water} matrices

The effect of different water matrices on the removal of DEET was evaluated using the $\mathrm{PMS} / \mathrm{CoSO}_{4}$ system (Fig. 2). The results indicated that the degradation efficiency of DEET in secondary wastewater and river water was slightly lower compared with that in ultrapure water. However, only $8 \%$ of DEET was successfully removed in seawater. It was suggested that the presence of a high concentration of the chloride ion in seawater had consumed most of the $\mathrm{SO}_{4}{ }^{-\cdot}$ to generate the chloride radical $(\mathrm{Cl})$. $\mathrm{Cl}^{\cdot}$ was then terminated through the formation of chlorine as indicated in Reactions (5)-(8) (Anipsitakis et al. 2006). Therefore, the application of $\mathrm{SO}_{4}{ }^{-\cdot}$ is not efficient in the treatment of DEET in seawater.

$\mathrm{SO}_{4}^{-\cdot}+\mathrm{Cl}^{-} \rightarrow \mathrm{SO}_{4}^{-\cdot}+\mathrm{Cl}^{-}$

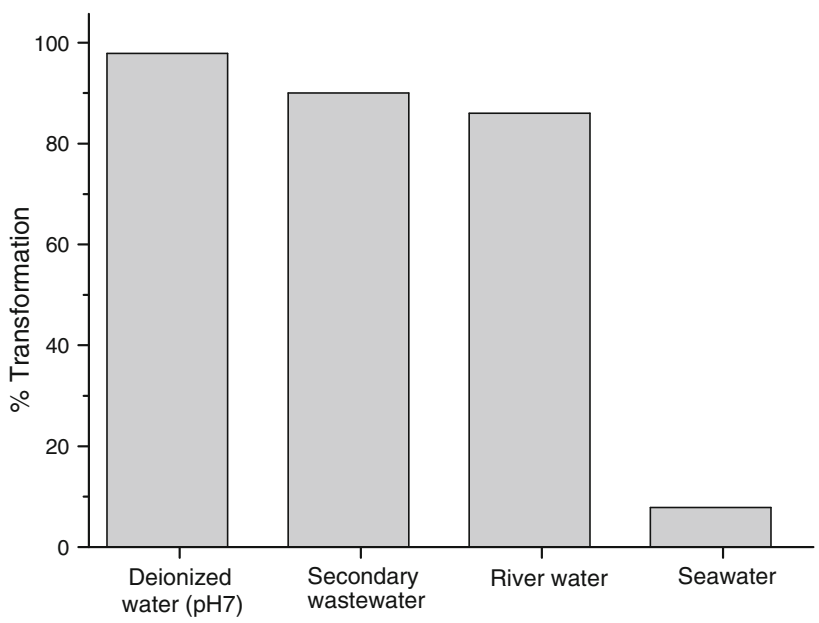

Fig. 2 Percentage of the removal of DEET in different water matrices using $\mathrm{Co}^{2+}$-PMS system

$\mathrm{Cl}^{\cdot}+\mathrm{Cl}^{-} \rightarrow \mathrm{Cl}_{2}^{-}$

$\mathrm{Cl}_{2}^{-\cdot}+\mathrm{Cl}_{2}^{-\cdot} \rightarrow \mathrm{Cl}_{2}+2 \mathrm{Cl}^{-}$

$\mathrm{Cl}+\mathrm{Cl}^{\cdot} \rightarrow \mathrm{Cl}_{2}$

Rate constants

The competition kinetics method has also been used to determine the rate constant for the reaction between $\mathrm{SO}_{4}{ }^{-}$. with caffeic acid (Swaraga and Adinarayana 2003) and carbamazepine (Matta et al. 2011). Basically, this method is based on the simultaneous oxidation of benzene (reference compound), whose rate constant for the reaction with $\mathrm{SO}_{4}{ }^{--}$is known, and DEET (target compound), whose rate constant for the reaction with $\mathrm{SO}_{4}{ }^{-\cdot}$ is to be determined. This method was validated using $p$-toluic acid, $m$-toluic acid and phenol. For $m$-toluic acid, $p$-toluic acid and phenol, the results indicated that the obtained rate constants are comparable with the values reported in previous studies (Table 1). The rate constant determined for the reaction between $\mathrm{SO}_{4}{ }^{-}$and DEET was $(1.9 \pm 0.1) \times 10^{9}$ $\mathrm{M}^{-1} \mathrm{~s}^{-1}$.

\section{Identification of transformation by-products}

GCMS analysis of the extracts from the chemically oxidized DEET solution indicates the generation of a variety of transformation by-products (Fig. 3).

Transformation by-products were identified based on their fragmentation in mass spectrum and also by comparing the mass spectrum data obtained with those previously reported in the literatures (Zhang and Lemley 2006; Tay et al. 2009; Medana et al. 2010) and also with synthesized reference compounds. As seen in Fig. 3 and 
Fig. 3 GCMS total ion chromatogram of DEET reaction extracts generated from a $\mathrm{FeSO}_{4}$-PMS, b $\mathrm{FeCl}_{2}$-PMS, c $\mathrm{CoSO}_{4}$-PMS and $\mathbf{d ~} \mathrm{CoCl}_{2}$-PMS

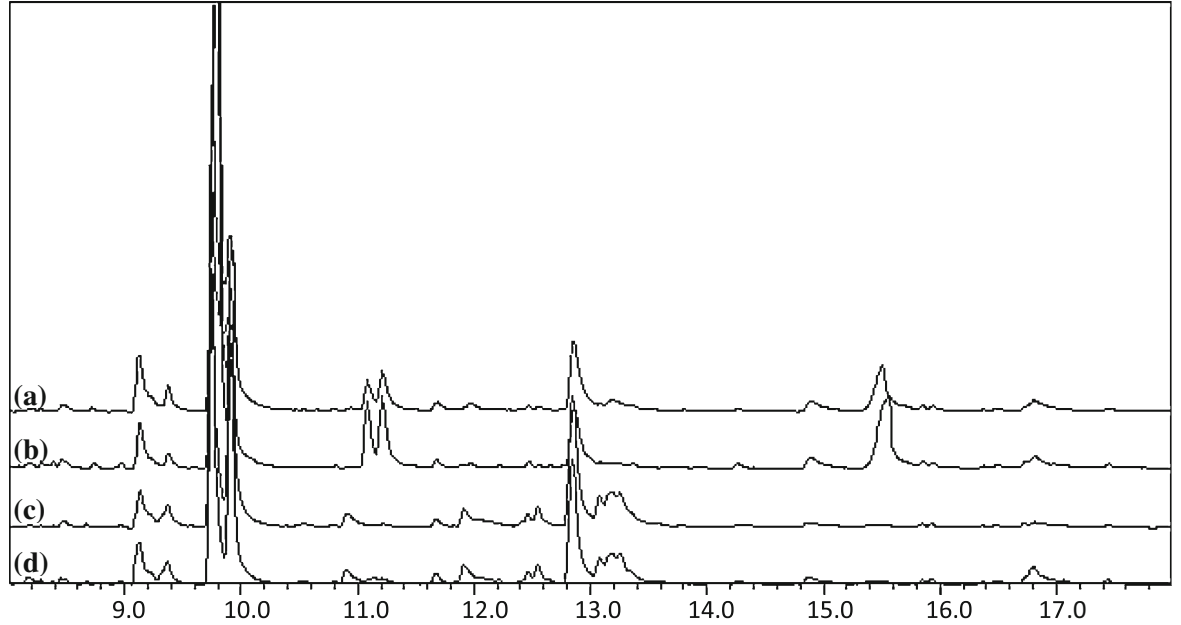

Table 2, there are slight differences in the transformation by-products of DEET that are generated using $\mathrm{Fe}^{2+}$ and $\mathrm{Co}^{2+}$ as activators. More transformation by-products are detected when the degradation of DEET is performed using $\mathrm{PMS} / \mathrm{Co}^{2+}$ systems. This might be due to the more reactive nature of PMS $/ \mathrm{Co}^{2+}$, where a higher amount of $\mathrm{SO}_{4}{ }^{-\cdot}$ can be produced to enhance the removal of DEET and its transformation by-products. Therefore, more transformation by-products can be generated using PMS $/ \mathrm{Co}^{2+}$ compared with $\mathrm{PMS} / \mathrm{Fe}^{2+}$.

The peak for DEET appears at the retention time of $9.78 \mathrm{~min}$. The fragmentation of DEET in electron impact mass spectrometry has been described in detail by Zhang and Lemley (2006) and Tay et al. (2009). The peak at $8.40 \mathrm{~min}$ represents $N, N$-diethylbenzamide $(\boldsymbol{A})$ with a molecular ion peak at $m / z, 177$ (Fig. S1a, Supplementary Data). $\boldsymbol{A}$ shows a base peak at $\mathrm{m} / \mathrm{z} 105$ formed through the elimination of the diethylamine group from the molecular ion. The ion at $\mathrm{m} / \mathrm{z} 77$ indicates the presence of a benzene ring.

The peaks at 9.10, 9.37 and 9.92 min show a base peak at $\mathrm{m} / \mathrm{z} 119$ representing the unmodified aromatic ring of DEET. The peaks at 9.10 and 9.37 min represent $N$-ethyl$m$-toluamide $(\boldsymbol{B})$ and $N, N$-dimethyl- $m$-toluamide $(\boldsymbol{C})$, respectively (Figs. S1b and c). $\boldsymbol{B}$ and $\boldsymbol{C}$ with a molecular weight of $163 \mathrm{amu}$ show almost a similar fragmentation pattern. B was confirmed by comparing its retention time with that of the synthesized reference compound. The peak at 9.92 is attributed to $N$-acetyl- $N$-ethyl- $m$-toluamide $(\boldsymbol{D})$. This compound shows a molecular ion peak at $\mathrm{m} / \mathrm{z} 205$ (Fig. S1d). The additional 14 amu compared with the molecular weight of DEET indicates the presence of a ketone group. The identity of $\boldsymbol{D}$ was also further confirmed by comparing its mass spectrum and retention time with that of the synthesized compound.
The peaks at $10.88,12.55$ and 13.25 min represent the isomers of monohydroxylated $N$-ethyl- $m$-toluamide (E1, $\boldsymbol{E 2}$, and $\boldsymbol{E 3})$. These compounds, with a molecular ion peak at $m / z$ 179, show a strong peak at $m / z 135$ (Figs. S1e-g). The addition of 16 amu compared with the peak at $\mathrm{m} / z 119$ in the mass spectrum of $\boldsymbol{B}$, indicates the presence of the additional $\mathrm{OH}$ group in the aromatic ring of $\boldsymbol{E} \mathbf{1}, \boldsymbol{E 2}$, and $\boldsymbol{E} 3$. The peaks at $11.08,11.21$ and $15.46 \mathrm{~min}$ are attributed to the isomers of monohydroxylated DEET $(\boldsymbol{F 1}, \boldsymbol{F} 2$, and F3) with a molecular ion peak at $\mathrm{m} / \mathrm{z}$ 206. The isomers of monohydroxylated DEET show a based peak at $\mathrm{m} / \mathrm{z} 135$ (Figs. S1 h-j). Dihydroxylated DEET $(\boldsymbol{G})$ with a molecular ion peak of $\mathrm{m} / \mathrm{z} 223$ has also been detected at $16.80 \mathrm{~min}$ (Fig. S1 k). For $\boldsymbol{G}$, the addition of $32 \mathrm{amu}$ indicates the addition of two $\mathrm{OH}$ groups to the aromatic ring of DEET.

The peaks at 12.84, 13.08 and $13.20 \mathrm{~min}$ are suggested to be 2-(diethylamino)-1- $m$-tolylethanone $(\boldsymbol{H}), \mathrm{N}$-ethyl- $\mathrm{N}$ (2-oxo-2- $m$-tolylethyl)acetamide $(\boldsymbol{J})$ and monohydroxylated 2-(ethyl(vinyl)amino)-1- $m$-tolylethanone $(\boldsymbol{K})$, respectively. The structure of these three compounds was exclusively proposed based on the fragmentation pattern in the mass spectrum. $\boldsymbol{H}, \boldsymbol{J}$, and $\boldsymbol{K}$ show a base peak or a strong peak at $m / z, 133$ (Figs. S1 $1-n$ ) representing the 2-oxo-2-m-tolylethan-1-ylium fragment, which can be stabilized by the presence of various resonance hybrids (Tay et al. 2009). A base peak at $m / z 150$ in the mass spectrum of $\boldsymbol{K}$ indicates the presence of the $\mathrm{OH}$ group at the aromatic ring of $\boldsymbol{H}$.

\section{Reaction mechanism}

According to the identified transformation by-products, both the aliphatic chain and the aromatic ring of DEET were found to react with $\mathrm{SO}_{4}{ }^{-\cdot}$ (Fig. 4). 
Table 2 DEET and its degradation by-products

\begin{tabular}{|c|c|c|c|c|c|c|}
\hline \multirow[t]{2}{*}{ Compound } & \multirow[t]{2}{*}{$\mathrm{RT}(\min )$} & \multirow{2}{*}{$\begin{array}{l}\text { Proposed structure of } \\
\text { degradation by-products }\end{array}$} & \multicolumn{4}{|c|}{ Oxidation system } \\
\hline & & & $\mathrm{PMS}_{-\mathrm{FeSO}}$ & PMS-FeCl 2 & PMS-CoSO 4 & PMS-CoCl ${ }_{2}$ \\
\hline DEET & 9.78 & & - & - & - & - \\
\hline A & 8.40 & & $\sqrt{ }$ & $\sqrt{ }$ & $\sqrt{ }$ & $\sqrt{ }$ \\
\hline$B$ & 9.10 & & $\sqrt{ }$ & $\sqrt{ }$ & $\sqrt{ }$ & $\sqrt{ }$ \\
\hline$C$ & 9.37 & & $\sqrt{ }$ & $\sqrt{ }$ & $\sqrt{ }$ & $\sqrt{ }$ \\
\hline$D$ & 9.92 & & $\sqrt{ }$ & $\sqrt{ }$ & $\sqrt{ }$ & $\sqrt{ }$ \\
\hline E1 & 10.88 & & & & $\sqrt{ }$ & $\sqrt{ }$ \\
\hline$E 2$ & 12.55 & & $\sqrt{ }$ & $\sqrt{ }$ & $\sqrt{ }$ & $\sqrt{ }$ \\
\hline E3 & 13.25 & & & & $\sqrt{ }$ & $\sqrt{ }$ \\
\hline$F 1$ & 11.08 & & $\sqrt{ }$ & $\sqrt{ }$ & & \\
\hline$F 2$ & 11.21 & & $\sqrt{ }$ & $\sqrt{ }$ & & \\
\hline$F 3$ & 15.46 & & $\sqrt{ }$ & $\sqrt{ }$ & & \\
\hline$G$ & 16.80 & & $\sqrt{ }$ & $\sqrt{ }$ & $\sqrt{ }$ & $\sqrt{ }$ \\
\hline$H$ & 12.84 & & $\sqrt{ }$ & $\sqrt{ }$ & $\sqrt{ }$ & $\sqrt{ }$ \\
\hline$J$ & 13.08 & & & & $\sqrt{ }$ & $\sqrt{ }$ \\
\hline$K$ & 13.20 & & & & $\sqrt{ }$ & $\sqrt{ }$ \\
\hline
\end{tabular}

The reaction at the aromatic ring of DEET was proposed to have started from the formation of the hexacyclodienyl radical cation (I) through the electron transfer from the aromatic ring to $\mathrm{SO}_{4}{ }^{-\cdot}$ (Anipsitakis et al. 2006). I can react with water to form the hydroxylated DEET radical (II). II could further react with oxygen to form radical III. Then, hydroxylated DEET $(\boldsymbol{F 1}, \boldsymbol{F} 2$ and $\boldsymbol{F 3})$ can be formed through the elimination of the peroxyl radical from III (Tay et al. 2010). Dihydroxylated DEET ( $\boldsymbol{G})$ can be formed through a further hydroxylation of monohydroxylated DEET. 


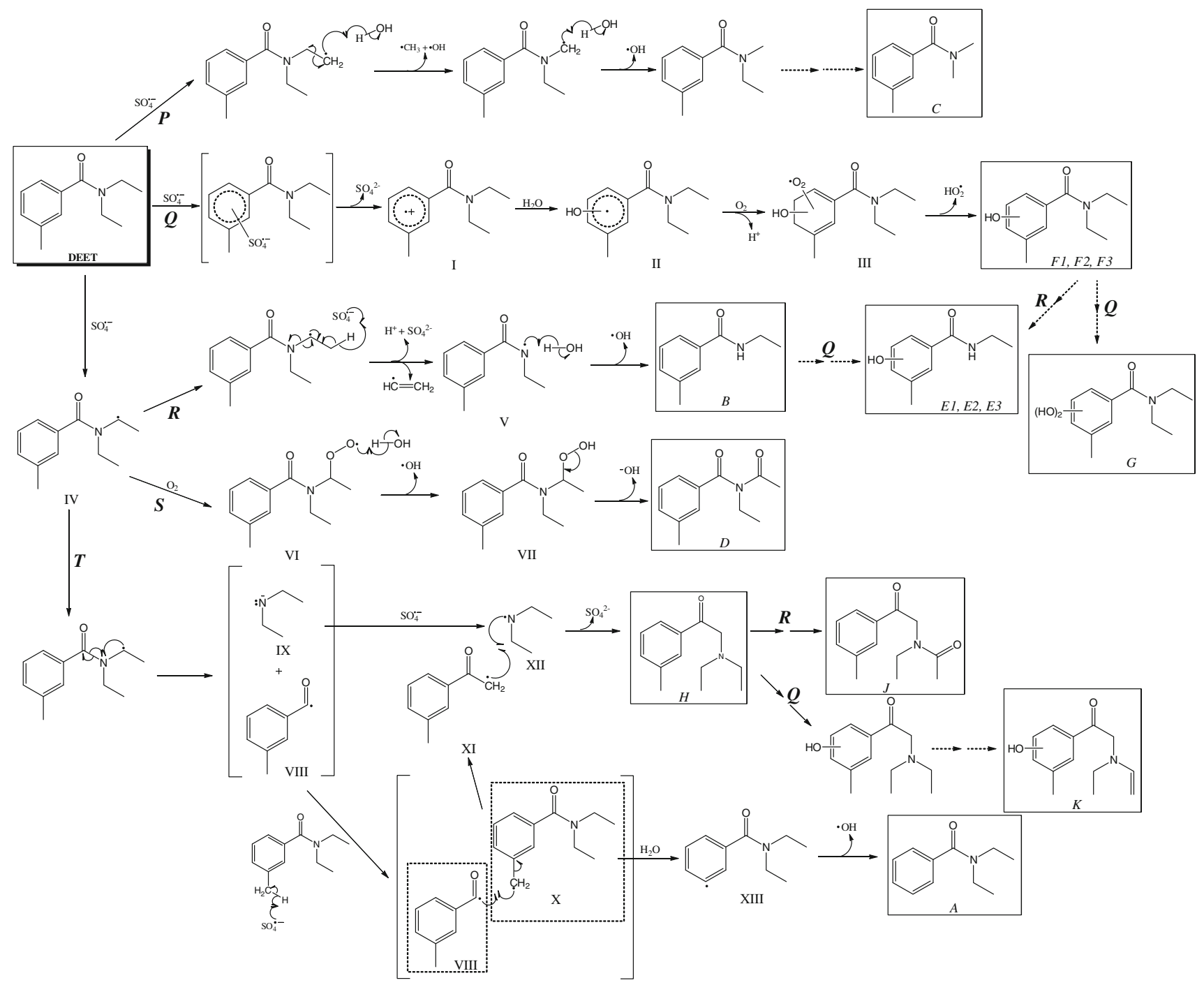

Fig. 4 Proposed reaction mechanism of DEET in $\mathrm{SO}_{4}{ }^{-\cdot}$-based oxidation

For the aliphatic chain of DEET, reactions are proposed to have started from radical IV. IV is suggested to be formed through the hydrogen abstraction at the ethyl group of DEET. Pathways $\boldsymbol{R}$ and $\boldsymbol{S}$ show the deethylation and oxidation of radical IV to form $\boldsymbol{B}$ and $\boldsymbol{D}$, respectively. The formation of B is proposed to have started from the deethylation of IV to form radical V. V then reacted with water to form $\boldsymbol{B} . \boldsymbol{E} 1, \boldsymbol{E} 2$, and $\boldsymbol{E} 3$ can also be formed through the hydroxylation of $\boldsymbol{B} . \boldsymbol{E 1}, \boldsymbol{E} 2$, and $\boldsymbol{E} 3$ may also be produced through the degradation of $\boldsymbol{F} \mathbf{1}, \boldsymbol{F} \mathbf{2}$, and $\boldsymbol{F 3}$, respectively, through pathway $\boldsymbol{R}$. On the other hand, IV can also react with oxygen to form the peroxyl radical VI. VI can then react with water to form radical VII, which later dissociate to form $\boldsymbol{D}$.

Besides the deethylation and oxidation, IV can be dissociated to form radical VIII and carbanion of diethylamine
(IX) via an intramolecular rearrangement. In addition, hydrogen abstraction of DEET can occur at the methyl group that is directly attached to the aromatic ring of DEET to form radical X (Tay et al. 2009). VIII can react with X to form radical $\mathrm{XI}$, which contains an additional $\mathrm{CH}_{2}$ group and also radical XIII. XI could then couple with a radical of diethylamine (XII) to form $\boldsymbol{H}$. $\boldsymbol{H}$ can be further transformed to $\boldsymbol{J}$ and $\boldsymbol{K}$. XIII can react with water to form $\boldsymbol{A}$, while $\boldsymbol{C}$ can be formed through the demethylation of DEET, as shown in Pathway $\boldsymbol{P}$.

\section{Conclusion}

The efficiency of DEET degradation using $\mathrm{SO}_{4}{ }^{-\cdot}$-based oxidation processes was evaluated. $\mathrm{SO}_{4}{ }^{-\cdot}$ was generated 
using $\mathrm{Co}^{2+}$ - and $\mathrm{Fe}^{2+}$-mediated activation of PMS. This result indicated that $\mathrm{Co}^{2+}$ is a better activator for PMS compared with $\mathrm{Fe}^{2+}$. Compared with $\mathrm{PMS} / \mathrm{Fe}^{2+}$, PMS/ $\mathrm{Co}^{2+}$ is also not influenced by counteranions. DEET removal was also found to be more favorable under an acidic condition. For the effect of water matrices, the results indicated that a high percentage $(>80 \%)$ of DEET removal can be achieved in river water and secondary wastewater using $\mathrm{SO}_{4}{ }^{-}$-based oxidation; however, this method is not efficient for the removal of DEET in seawater $(\sim 8 \%)$. The second order rate constant for the reaction of $\mathrm{SO}_{4}{ }^{-\cdot}$ with DEET was $(1.9 \pm 0.1) \times 10^{9}$ $\mathrm{M}^{-1} \mathrm{~s}^{-1}$. In this study, 14 transformation by-products of DEET generated using the combination of $\mathrm{PMS} / \mathrm{FeSO}_{4}$, $\mathrm{PMS} / \mathrm{FeCl}_{2}, \mathrm{PMS} / \mathrm{CoSO}_{4}$, and $\mathrm{PMS} / \mathrm{CoCl}_{2}$ systems have been identified.

Acknowledgments This research was financially supported by Ministry of Higher Education of Malaysia (FRGS FP018-2008C) and University of Malaya (PPP PS354-2010A).

\section{References}

Anipsitakis GP, Dionysiou DD, Gonzalez MA (2006) Cobaltmediated of peroxymonosulfate and sulfate radical attack on phenolic compounds-implications of chloride ions. Environ Sci Technol 40:1000-1007

Chan KH, Chu W (2009) Degradation of atrazine by cobalt-mediated activation of peroxymonosulfate: different cobalt counteranions in homogenous process and cobalt oxide catalysts in photolytic heterogeneous process. Water Res 43:2513-2521

Chan WT, Graham NJD, Chu W (2010) Degradation of iopromide by combined UV irradiation and peroxydisulfate. J Hazard Mater 181:508-513

Fradin MD (1998) Mosquitoes and mosquito repellents: a clinician's guide. Ann Intern Med 128:931-940

Kolpin DW, Skopec M, Meyer MT, Furlong ET, Zaugg SD (2004) Urban contribution of pharmaceuticals and other organic wastewater contaminants to streams during differing flow conditions. Sci Total Environ 328:119-130

Liang C, Guo YY, Chieng YC, Wu YJ (2010) Oxidative degradation of MTBE by pyrite-activated persulfate: proposed reaction pathways. Ind Eng Chem Res 49:8858-8864

Manoj P, Prasanthkumar KP, Manoj WM, Aravind UK, Manojkumar TK, Aravindakumar CT (2007) Oxidation of substituted triazines by sulfate radical anion $\left(\mathrm{SO}_{4}{ }^{-}\right)$in aqueous medium: a laser flash photolysis and steady state radiolysis study. J Phys Org Chem 20:122-129

Matta R, Tlili S, Chiron S, Barbati S (2011) Removal of carbamazepine from urban wastewater by sulfate radical oxidation. Environ Chem Lett 9:347-353

McKay G, Dong MM, Kleinmant JL, Mezykt SP, Rosario-Ortiz FL (2011) Temperature dependence of the reaction between the hydroxyl radical and organic matter. Environ Sci Technol 45:6932-6937

Medana C, Calza P, Bello FD, Raso E, Minero C, Baiocchi C (2010) Multiple unknown degradants generated from the insect repellent
DEET by photoinduced processes on $\mathrm{TiO}_{2}$. J Mass Spectrom 46:24-40

Méndez-Díaz J, Sánchez-Polo M, Rivera-Utrilla J, Canonica S, von Gunten U (2010) Advanced oxidation of the surfactant SDBS by means of hydroxyl and sulphate radicals. Chem Eng J 163:300306

Neta P, Madhavan V, Zemel H, Fessenden RW (1977) Rate constants and mechanism of reaction of $\mathrm{SO}_{4}{ }^{-\cdot}$ with aromatic-compounds. J. Am. Chem. Soc. 99:163-164

Nfodzo P, Choi H (2011) Sulfate radicals destroy pharmaceuticals and personal care products. Environ Eng Sci 28:605-609

Rastogi A, Al-Abed SR, Dionysiou DD (2009) Sulfate radical-based ferrous-peroxymonosulfate oxidative system for PCBs degradation in aqueous and sediment systems. Appl Catal B-Environ 85:171-179

Rickman KA, Mezyk SP (2010) Kinetics and mechanisms of sulfate radical oxidation of $\beta$-lactam antibiotics in water. Chemosphere 81:359-365

Stackelberg PE, Furlong ET, Meyer MT, Zaugg SD, Henderson AK, Reissman DB (2004) Persistence of pharmaceutical compounds and other organicwastewater contaminants in a conventional drinking-water-treatment plant. Sci Total Environ 329:99-113

Stasinakis AS (2008) Use of selected advanced oxidation processes (AOPs) for wastewater treatment-a mini review. Global Nest J 10:376-385

Swaraga MS, Adinarayana M (2003) Kinetics and mechanism of protection of adenine from sulphate radical anion by caffeic acid under anoxic conditions. Indian $\mathrm{J}$ Biochem Biophys 40:27-30

Tay KS, Rahman NA, Abas MRB (2009) Degradation of DEET by ozonation in aqueous solution. Chemosphere 76:12961302

Tay KS, Rahman NA, Abas MRB (2010) Ozonation of parabens in aqueous solution: Kinetics and mechanism of degradation. Chemosphere 81:1446-1453

Vahabzadeh MAF, Bonakdarpour B, Mofarrah E, Mehranian M (2005) Application of the central composite design and response surface methodology to the advanced treatment of olive oil processing wastewater using Fenton's peroxidation. J Hazard Mater 123:187-195

Wang D, Li Y, Yang M, Han M (2008) Decomposition of polycyclic aromatic hydrocarbons in atmospheric aqueous droplets through sulfate anion radicals: an experimental and theoretical study. Sci Total Environ 393:64-71

Weigel S, Kuhlmann J, Hühnerfuss H (2002) Drugs and personal care products as ubiquitous pollutants: occurrence and distribution of clofibric acid, caffeine and DEET in the North Sea. Sci Total Environ 295:131-141

Weigel S, Berger U, Jensen E, Kallenborn R, Thoresen H, Hühnerfuss $\mathrm{H}$ (2004) Determination of selected pharmaceuticals and caffeine in sewage and seawater from Troms $\varnothing /$ Norway with emphasis on ibuprofen and its metabolites. Chemosphere 56:583-592

Weinberg HS, Pereira VJ, Ye Z (2008) Drugs in drinking water: treatment options. In: Aga DS (ed) Fate of pharmaceuticals in the environment in water treatment systems. CRC Press, New York, pp 217-227

Yang Q, Choi H, Chen Y, Dionysiou DD (2008) Heterogeneous activation of peroxymonosulfate by supported cobalt catalyst for the degradation of 2,4-dichlorophenol in water: the effect of support, cobalt precursor, and UV radiation. Appl Catal B-Environ 77:300-307

Zhang H, Lemley AT (2006) Reaction mechanism and kinetic modeling of DEET degradation by flow through anodic Fenton treatment (FAFT). Environ Sci Technol 40:4488-4494 
Zhang H, Lemley AT (2007) Evaluation of the performance of flow through anodic Fenton treatment in amide compound degradation. J Agric Food Chem 55:4073-4079
Ziajka J, Pasiuk-Bronikowska W (2005) Rate constants for atmospheric trace organic scavenging $\mathrm{SO}_{4}{ }^{-}$in the Fe-catalysed autoxidation of S(IV). Atmos Environ 39:1431-1438 